\title{
INTERACTION OF GRAVITATIONAL RADIATION WITH A UNIFORMLY MAGNETIZED SPHERE
}

\author{
V. DE SABBATA, P. FORTINI, and C. GUALDI \\ Istituto di Fisica dell'Università Bologna, Bologna, Italy \\ and \\ L. FORTINI BARONI
}

Holder of a C.N.R. 'Italian National Research Council' Scholarship, Bologna, Italy

\begin{abstract}
Maxwell equations in the field of a gravitational wave are linearized by means of the weak field approximation. Then the equations are solved in the case of a uniformly magnetized sphere and the dipole electromagnetic radiation power is calculated. These results are applied to compute the electromagnetic radiation emitted by magnetic neutron stars and by the Earth when hit by gravitational radiation.
\end{abstract}

